\title{
Le laser pour redonner leurs couleurs aux peintures murales
}

L'application des techniques laser à la conservation-restauration des objets du patrimoine s'est largement développée au cours des dernières décennies. Les méthodes d'analyse et d'imagerie par faisceau laser, en améliorant la connaissance des matériaux et de leurs mécanismes d'altération, apportent des informations déterminantes pour une meilleure conservation des œuvres. Par ailleurs, les performances accrues des lasers pulsés ont permis le développement de techniques de nettoyage adaptées à la restauration d'objets complexes et fragiles, tels que les sculptures et éléments d'ornement ou les peintures sur toile [1, 2]. Une nouvelle application laser vient d'être mise au point pour répondre à une problématique de conservation des peintures et décors muraux connue depuis plusieurs siècles : redonner leur couleur rouge aux pigments à base de plomb qui noircissent au fil des ans.

Sébastien AZE
Philippe DELAPORTE
Laboratoire Lasers, plasmas et procédés
photoniques - UMR 6182 CNRS, Marseille
Jean-Marc VALLET
Centre interrégional de conservation
et restauration du patrimoine, Marseille
Vincent DETALLE
Laboratoire de recherche des monuments
historiques, Champs-sur-Marne
Olivier GRAUBY
Centre de recherche en matière
condensée et nanosciences -
UPR 7251 CNRS, Marseille
aze@lp3.univ-mrs.fr

\section{Le noircissement du minium dans les œuvres d'art}

Les pigments à base de plomb, en particulier le minium de couleur rouge $\mathrm{Pb}_{3} \mathrm{O}_{4}$, sont connus pour leur tendance à noircir, ce qui modifie drastiquement l'aspect et l'interprétation des œuvres. Ce phénomène est particulièrement prononcé en peinture murale, lorsque le minium a été appliqué à une fresque (figure 1); et de nombreuses œuvres présentant un grand intérêt historique et patrimonial sont affectées par ce type d'altération [3].

Le noircissement du minium est généralement causé par la transformation du pigment en plattnérite $\beta-\mathrm{PbO}_{2}$, comme l'atteste l'analyse de microprélèvements de matière picturale [4].

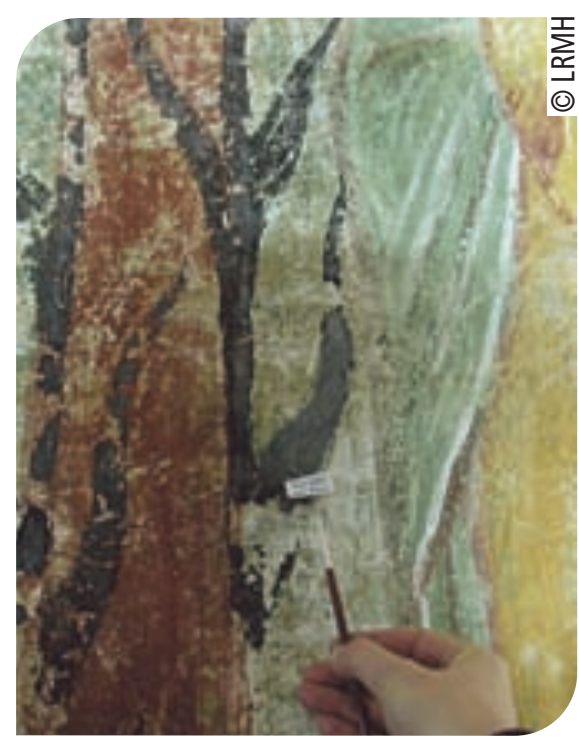

Figure 1. « Les flammes de l'Enfer », peintures murales de l'Abbaye de Saint-Savin-surGartempe (Vienne, xII ${ }^{e}$ siècle). Le noircissement des flammes, peintes au minium, est attribué à la transformation du pigment en plattnérite.

Les études les plus récentes ont mis en évidence l'influence de facteurs environnementaux (humidité, température, pollutions gazeuses) sur l'apparition et le développement de cette phase d'altération [5]. À l'issue du processus de transformation, la couche picturale à base de minium peut être entièrement transformée en plattnérite (figure 2). Jusqu'à présent, ce phénomène a été considéré comme irréversible, aucune méthode de restauration ne permettant de restituer l'état d'origine de la couche picturale.
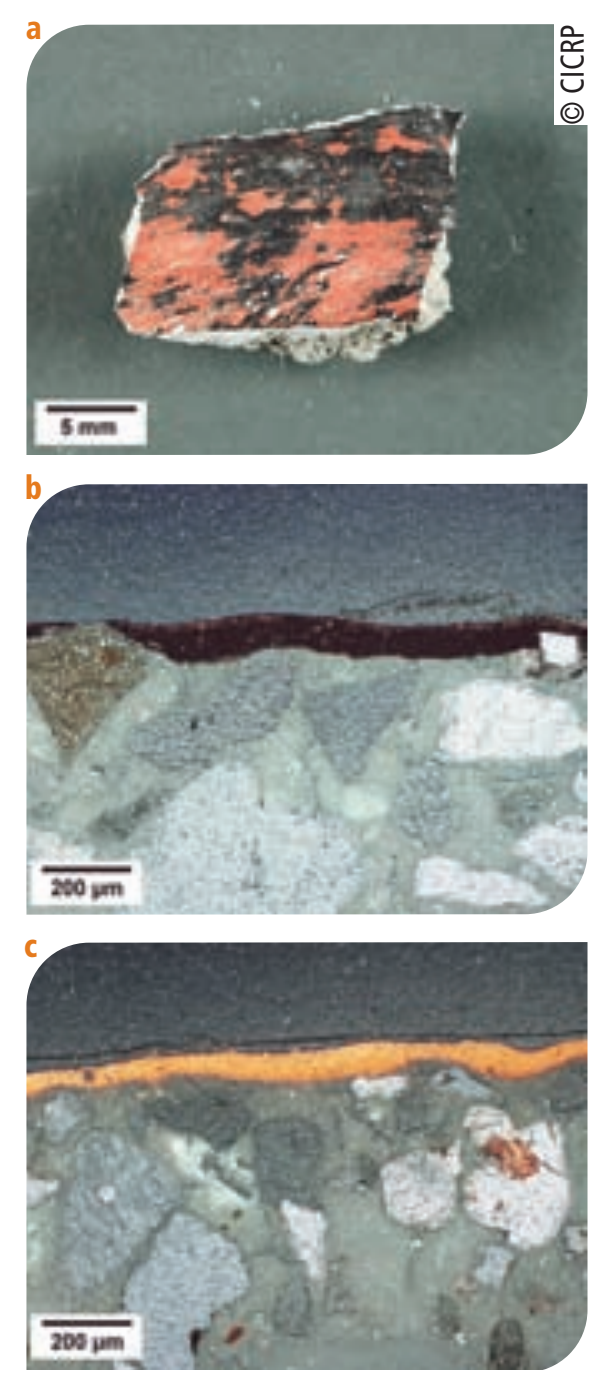

Figure 2. Fragment de peinture murale dans laquelle la couche de minium a été partiellement transformée en plattnérite (a). L'altération est hautement hétérogène, avec des zones où la couche picturale est intégralement noircie (b) et d'autres où la couche picturale est intacte (c). 


\section{Irradier pour restaurer}

\section{Repasser de la plattnérite au minium...}

L'étude de la réduction de la plattnérite par voie thermique a mis en évidence la possible reconversion de la plattnérite en minium [6]. En effet, le minium est formé spontanément par un traitement thermique de la plattnérite à $375^{\circ} \mathrm{C}$. Cette propriété pourrait être mise à profit pour restituer aux couches de minium noirci leur composition et leur couleur d'origine. Cependant, une telle technique nécessite que des conditions spécifiques soient remplies :

- I'apport de chaleur doit être localisé, afin que seules les zones noircies soient effectivement soumises à l'échauffement ;

- la plattnérite doit effectivement être transformée en minium, sans risque de formation d'autres phases ;

- il doit être possible de faire un traitement in situ, notamment pour les peintures murales.

\section{...par irradiation laser}

Ces contraintes ont mené au choix de I'utilisation d'un faisceau laser continu comme source ponctuelle de chaleur. Différentes sources laser ont été sélectionnées afin d'optimiser les conditions d'irradiation (longueur d'onde, puissance, temps d'irradiation, profil du faisceau...). Les sources laser ont été choisies dans les longueurs d'onde visible ou proche infrarouge : laser à argon ionisé $\left(\mathrm{Ar}^{+}\right)$, émettant à $488 \mathrm{~nm}$, laser fibré à diode Ga:As, émettant à $808 \mathrm{~nm}$, et laser Nd:YAG continu, émettant à $1064 \mathrm{~nm}$.

Des essais préliminaires ont été réalisés sur des échantillons homogènes de plattnérite pure en poudre pour déterminer les seuils de puissance entraînant la formation du minium, indépendamment de la structure de la couche de plattnérite et de la nature du substrat. Puis, pour affiner ces seuils et prendre en considération la complexité des matériaux dans les cas réels d'altération, des essais d'irradiation ont été menés sur des fragments prélevés sur des peintures murales expérimentales, réalisées en 1982 à l'initiative du Laboratoire de recherche des monuments historiques [7], qui ont subi un vieillissement naturel ayant entraîné la transformation du minium en plattnérite.

Pour chaque source laser, les transformations induites par l'irradiation ont été étudiées en fonction de la densité de puissance déposée et du temps d'irradiation. La structure et la composition de la couche picturale avant et après traitement ont été déterminées à l'aide de méthodes d'observation (microscopie optique, microscopie électronique à balayage) et d'analyse (diffraction de rayons $X$, microspectrométrie Raman).

\section{Optimisation des conditions d'irradiation}

\section{Laser à argon ionisé (488 nm)}

Les essais réalisés avec le laser $\mathrm{Ar}^{+}$montrent que du minium est formé rapidement sous le faisceau lorsqu'une faible puissance est appliquée, dès $12 \mathrm{~W} . \mathrm{cm}^{-2}$. Néanmoins, la transformation n'est effective que sur une partie superficielle de la couche picturale.

L'augmentation de la densité de puissance déposée entraîne, dès $14 \mathrm{~W} . \mathrm{cm}^{-2}$, la formation d'une nouvelle phase plombifère, de couleur jaune, au centre de la zone irradiée (figure 3a). Les analyses ponctuelles par microspectrométrie Raman (figure 3b) montrent qu'il s'agit de massicot, la variété ortho-

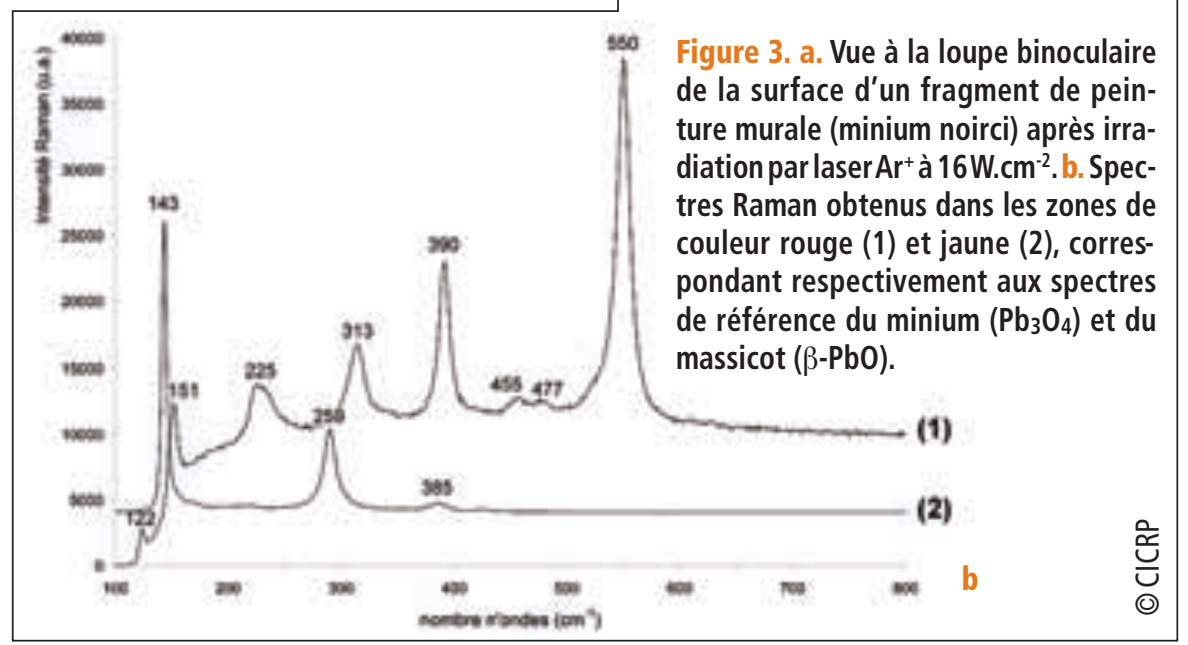

rhombique du monoxyde de plomb $\beta$-PbO. La formation de massicot montre un échauffement excessif de la couche picturale à une température supérieure à $512^{\circ} \mathrm{C}[8]$.

\section{Laser Nd:YAG (1064 nm)}

Avec le laser Nd:YAG, le minium est formé sur une large gamme de puissance. Ainsi, les premiers grains de minium sont formés lorsque l'énergie déposée dépasse $8 \mathrm{~W} . \mathrm{cm}^{-2}$, la proportion de minium formé augmentant avec la puissance. Au-dessus d'environ $100 \mathrm{~W} . \mathrm{cm}^{-2}$, les observations montrent la reconversion de la plattnérite sur toute l'épaisseur de la couche picturale, les premiers grains de massicot n'étant formés $\mathrm{qu}^{\prime}$ au-dessus de $200 \mathrm{~W} . \mathrm{cm}^{-2}$.

\section{Laser Ga:As fibré (808 nm)}

Les essais menés sur les fragments de peinture murale montrent la possibilité de transformation de $\beta-\mathrm{PbO}_{2}$ en $\mathrm{Pb}_{3} \mathrm{O}_{4}$ dans une gamme de densité de puissance comprise entre 23 et 43 W.cm ${ }^{-2}$.

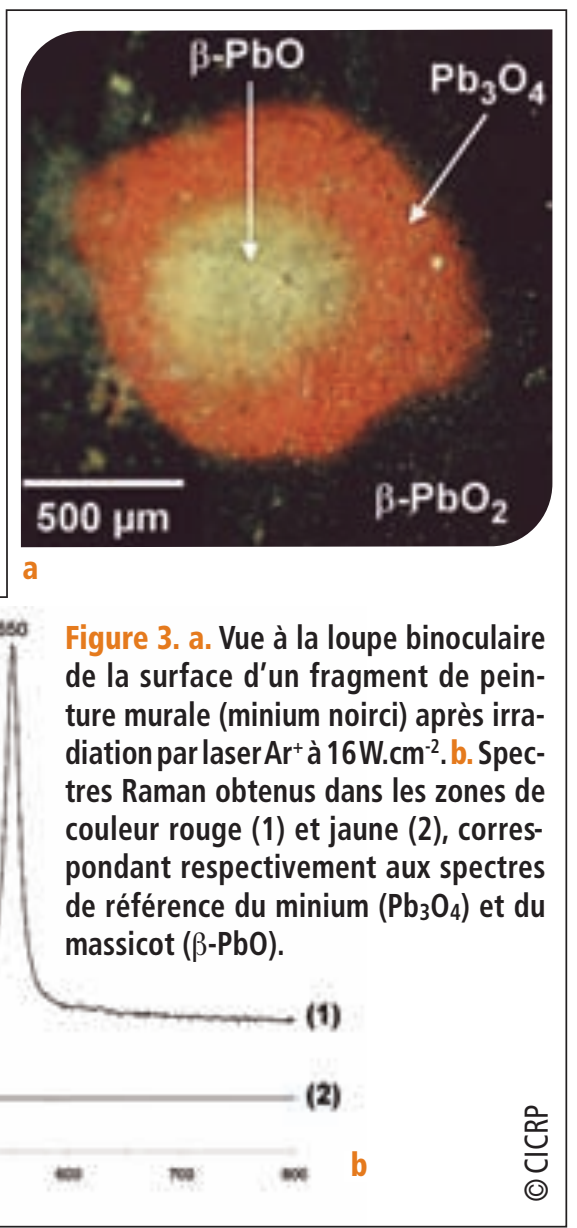




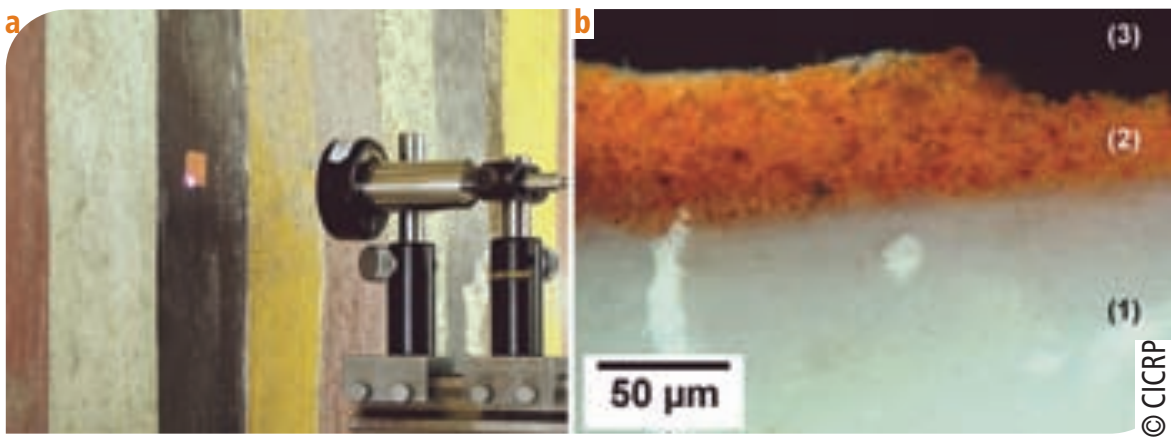

Figure 4. a. Essais d'irradiation in situ de peintures murales expérimentales contenant du minium noirci. Le faisceau délivré par la source laser (diode Ga:As fibrée) est mis en forme par un dispositif optique dont les déplacements permettent le balayage de la zone à traiter. b. Observation en microscopie optique d'un échantillon de peinture murale après irradiation au laser fibré à diode Ga:As $\left(35 \mathrm{~W} . \mathrm{cm}^{-2}\right)$ préparé sous forme de section polie : enduit sous-jacent à base de chaux (1), couche de minium (2), résine d'enrobage (3).

Ces essais préalables ont permis de traiter des zones étendues, par balayage du faisceau sur plusieurs centimètres carrés. Des essais in situ ont également été réalisés sur les peintures murales expérimentales dans des zones peintes selon la technique de la fresque (figure 4a) ; I'observation des prélèvements effectués après traitement, préparés sous la forme de sections polies, montre que la reconversion est effective sur toute l'épaisseur de la couche picturale (figure 4b).

\section{Conclusion}

Les essais $d$ 'irradiation réalisés avec différentes sources laser et sur différents types d'échantillons ont permis de valider une méthode innovante pour resti-

\section{Références}

[1] P. Pouli, D.C. Emmony. «The effect of ND:YAG laser radiation on medieval pigments». Journal of Cultural Heritage 1 (2000) S181-S188.

[2] M. Castillejo, M. Martin, M. Oujja, D. Silva, R. Torres. "Analytical study of the chemical and physical changes induced by KrF laser cleaning of tempera paints». Analytical Chemistry 74 (2002) 4462-4471.

[3] S. Aze, J.-M. Vallet, V. Detalle, 0. Grauby, A. Baronnet. "Chromatic alterations of red lead pigments in artworks: a review». Phase Transitions 81 2-3 (2008) 145-154.

[4] S. Daniilia, E. Minopoulou, D. Demosthenou, G. Karagianis. "A comparative study of wall paintings at the Cypriot monastery of Christ Antiphonitis: one artist or two?». Journal of Archaeological Science 35 (2008) 1695-1707. tuer la composition et la couleur originelle des peintures au minium ayant subi un noircissement : I'utilisation d'une source fibrée émettant dans le proche infrarouge (diode Ga:As, 808 $\mathrm{nm}$ ) rend possible l'application in situ de cette technique et minimise les risques d'échauffement excessif de la couche picturale (et donc les risques de formation de phases plombifères autres que le minium).

Un premier traitement a été effectué sur une peinture murale du $x \mathrm{xx}^{\mathrm{e}}$ siècle ornant la chapelle de Solomiat (Ain), qui présentait de grandes surfaces de minium noirci. Cette mise en pratique concluante permet d'envisager l'application de cette technique à d'autres œuvres présentant cette forme d'altération.

[5] S. Aze, J.-M. Vallet, M. Pomey, A. Baronnet, 0 . Grauby. « Red lead darkening in wall paintings: natural ageing of experimental wall paintings versus artificial ageing tests». European Journal of Mineralogy 196 (2007) 883-890.

[6] S. Aze, V. Detalle, J.-M. Vallet, N. Pingaud. «L'altération des pigments au plomb : étude du minium et de sa possible reconversion ». L'Actualité chimique 318 (2008), 9-15.

[7] A. Morineau, M. Stefanaggi. « Statistical approach to the problem of frescoes: the French experience ». Journal of the Italian Statistical Society, 4 (1995) 37-53.

[8] D.A. Ciomartan, R.J.H. Clark, L.J. McDonald, M. Odlyha. "Studies on the thermal decomposition of basic lead (II) carbonate by Fourier-transform Raman spectroscopy, X-ray diffraction, and thermal analysis». Journal of Chemical Society: Dalton Transactions 18 (1996) 3639-3645.

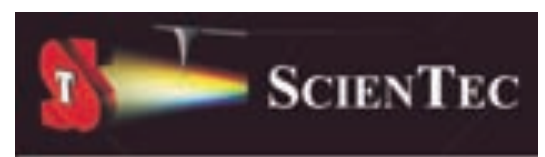

\section{Nano-positionnement}

RUS DE REECCSNOY AFC VOTRE AFM

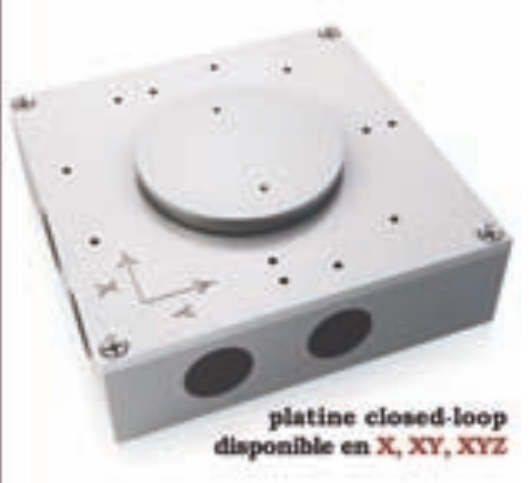

Différentes gammes de balayage :

- Zoem en un seul clic

- Repositionaemeat en an seul elie

- Pas de dérive da pieno

- Balayage linèaire

- Prédisian de mesure

Exemples d'applications :

- Lithographie

- Manipulation à réchelle nanométrique

- Mttrologie

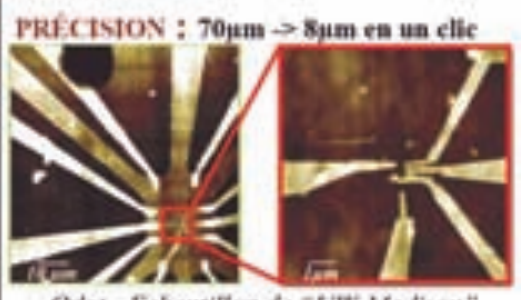

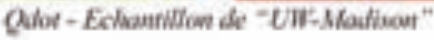

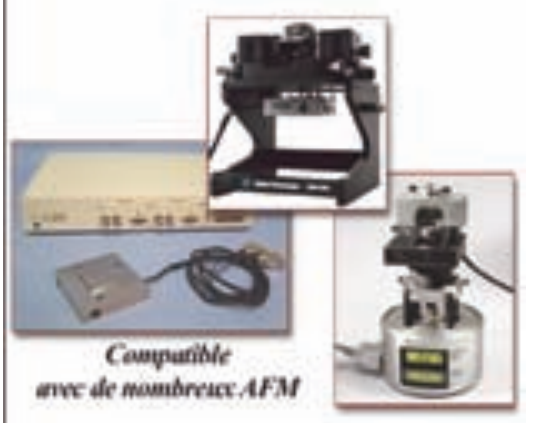

www.scientec.fr

Têléphane : 01.64.53.27.00 